DESY 98-104

August 1998

\title{
Degenerate Dirac Neutrinos
}

\author{
$\mathrm{UTPAL}_{\text {SARKAR }}^{(a, b)}$ 円 \\ (a) Theory Group, DESY, Notkestraße 85, 22607 Hamburg, Germany \\ ${ }^{(b)}$ Theory Group, Physical Research Laboratory, Ahmedabad, 380 009, India \&
}

\begin{abstract}
A simple extension of the standard model is proposed in which all the three generations of neutrinos are Dirac particles and are naturally light. We then assume that the neutrino mass matrix is diagonal and degenerate, with a few $\mathrm{eV}$ mass to solve the dark matter problem. The self energy radiative corrections, however, remove this degeneracy and allow mixing of these neutrinos. The electroweak radiative corrections then predict a lower bound on the $\nu_{\mu}-\nu_{e}$ mass difference which solves the solar neutrino problem through MSW mechanism and also predict a lower bound on the $\nu_{\tau}-\nu_{\mu}$ mass difference which is just enough to explain the atmospheric neutrino problem as reported by super Kamiokande.
\end{abstract}

\footnotetext{
${ }^{1}$ E-mail: utpal@prl.ernet.in

${ }^{2}$ permanent address
} 
The neutrinoless double beta decay [1] puts severe constraints on the Majorana mass of the $\nu_{e}$. With this constraint it is not possible to explain simultaneously the dark matter problem [2], solar neutrino problem [3], atmospheric neutrino problem [4] and the laboratory bounds on the mixing angles [5]. Since the preliminary results from KARMEN [6] contradicts the LSND result [6], we shall not include that in our analysis. If the neutrinos are Dirac particle there will not be any lepton number violation and hence there will not be any constraint from the neutrinoless double beta decay. In that case one can postulate an almost degenerate neutrino scenario [8] to explain the other problems.

In this article, we consider exactly degenerate Dirac particles with a few $\mathrm{eV}$ mass to explain the dark matter problem. However, this will not allow any flavour mixing. So we introduce explicit lepton flavour violation, which will break the mass degeneracy radiatively, which in turn will allow flavour mixing. The electroweak self-energy corrections will then predict a lower bound on the mass squared differences between $\nu_{\mu}$ and $\nu_{e}$ which can solve the solar neutrino problem through matter enhanced neutrino oscillation [9] and simultaneously predict a lower bound on the mass squared difference between $\nu_{\tau}$ and $\nu_{\mu}$ which is just enough to solve the atmospheric neutrino problem. There is also a similar contribution to mass splitting from flavour violating radiative corrections, which also gives the maximal neutrino flavour mixing.

Consider a two generation Majorana neutrino scenario. The neutrino mass matrix is given by,

$$
M_{\nu}=\left(\begin{array}{cc}
m_{e e} & m_{e \mu} \\
m_{\mu e} & m_{\mu \mu}
\end{array}\right) .
$$

The neutrinoless double beta decay [1] will imply $m_{e e}<0.46 \mathrm{eV}$. If we consider an almost degenerate neutrino scenario [8], to solve the dark matter problem [2] we require $m_{\mu \mu} \simeq m_{e e}$. Then the small mixing [5] of $\nu_{e}$ with $\nu_{\mu}$ will imply that $\sin ^{2} \theta_{e \mu} \sim \frac{m_{e \mu}}{m_{\mu \mu}}<0.6$ and the masses of the $\nu_{e}$ and $\nu_{\mu}$ are 
less than $1 \mathrm{eV}$. This will not solve the dark matter problem. This problem is solved if one assumes that the neutrinos are Dirac particles. However, the main problem of making the neutrinos a Dirac particle is that, in a simple extension of the standard model the Dirac mass of the neutrinos are related to the charged lepton masses and hence cannot be small (of the order of a few eV) naturally.

We now propose a scenario where the neutrinos are Dirac particles and are naturally light. The left handed neutrinos combine with the right handed neutrinos through their interactions with a different higgs doublet, which does not couple to the quarks and charged leptons because of the presence of an additional $U(1)$ symmetry. This new higgs doublet acquires a small vacuum expectation value (vev), when the extra $U(1)$ symmetry and the electroweak symmetry are broken [10], and hence its coupling gives a small Dirac mass to the neutrinos naturally. There is no lepton number violation in this scenario and hence there are no Majorana mass of the neutrinos.

We extend the standard model gauge group to include a new $U(1)$ symmetry,

$$
\mathcal{G}_{\text {ext }} \equiv S U(3)_{c} \times S U(2)_{L} \times U(1)_{Y} \times U(1)_{X}
$$

We also extend the model to include the three right handed neutrinos $\nu_{i R}$ $(i=1,2,3)$; four additional singlet fermions $Y_{i}(i=1,2,3)$ and $Z$, which are required for purpose of anomaly cancellation; one new higgs doublet $\eta$, a scalar singlet $\chi$ and a charged scalar singlet $\zeta$. Transformation properties of the new particles are presented in table 1 . The scalar $\chi$ acquires a vev at a very high scale $\mathrm{M}$, breaks the $U(1)_{X}$ symmetry and give masses to the extra singlet fields. The mass of the doublet $\eta$ is also of the order of M, but it does not acquire any vev to start with. However, after the electroweak symmetry breaking it acquires a small vev and gives small Dirac masses to the neutrinos naturally.

The lagrangian contains the quadratic and the quartic terms and the 
Table 1: transformations of the new particles

\begin{tabular}{||l|c||}
\hline \hline & Fermions \\
\hline$\nu_{i R}$ & $(1,1,0,2)$ \\
\hline$Y_{i}$ & $(1,1,0,-1)$ \\
\hline$Z$ & $(1,1,0,-3)$ \\
\hline \hline & Scalars \\
\hline$\eta$ & $(1,2,-1 / 2,-2)$ \\
\hline$\chi$ & $(1,1,0,2)$ \\
\hline$\zeta$ & $(1,1,1,-2)$ \\
\hline \hline
\end{tabular}

trilinear mixing terms which are given by,

$$
\begin{aligned}
\mathcal{L}_{\text {scalar }} & =M_{\eta}^{2} \eta^{\dagger} \eta+M_{\chi}^{2} \chi^{\dagger} \chi+m_{\phi}^{2} \phi^{\dagger} \phi \\
& +\frac{1}{2} \lambda_{1}\left(\phi^{\dagger} \phi\right)^{2}+\frac{1}{2} \lambda_{2}\left(\eta^{\dagger} \eta\right)^{2}+\frac{1}{2} \lambda_{3}\left(\chi^{\dagger} \chi\right)^{2} \\
& +\frac{1}{2} \lambda_{4}\left(\phi^{\dagger} \phi\right)\left(\eta^{\dagger} \eta\right)+\frac{1}{2} \lambda_{5}\left(\chi^{\dagger} \chi\right)\left(\eta^{\dagger} \eta\right)+\frac{1}{2} \lambda_{6}\left(\phi^{\dagger} \phi\right)\left(\chi^{\dagger} \chi\right) \\
& +\mu \phi^{\dagger} \eta \chi .
\end{aligned}
$$

The scalar $\chi$ acquires a vev at some large scale $M$, which is the only other mass scale in the model other than the electroweak symmetry breaking scale. We consider

$$
M_{\chi} \sim M_{\eta} \sim<\chi>\sim M \sim 10^{11} \mathrm{GeV} .
$$

Then to prevent the usual higgs doublet from acquiring a large mass we require

$$
\mu \sim m_{\phi} \sim m
$$

where $m$ is the electroweak syummetry breaking scale.

We may now minimize the potential to determine the vevs of the different scalar fields. They are given by,

$$
<\chi>^{2} \sim-\frac{M_{\chi}^{2}}{\lambda_{3}} ; \quad<\phi>^{2} \sim \frac{-\lambda_{1} m_{\phi}^{2}+\lambda_{4} M_{\eta}^{2}}{\lambda_{1} \lambda_{2}-\lambda_{4}^{2}}
$$




$$
\text { and } \quad<\eta>\sim \frac{\mu<\phi><\chi>}{M_{\eta}^{2}} \sim 100 \mathrm{eV}
$$

The scalar field $\eta$ acquires a very small vev naturally in this scenario. As a result, if it gives a Dirac mass to the neutrinos, then we have a natural explanation of the smallness of the Dirac mass of the neutrinos. To obtain the mass of the neutrinos we now write down the Yukawa couplings of the leptons and the singlet fermions,

$$
\mathcal{L}=f_{\nu i \alpha} \overline{l_{i L}} \nu_{\alpha R} \eta+g_{a b} \overline{Y_{a}^{c}} Y_{b} \chi+g_{a}^{\prime} \overline{Y_{a}} Z \chi+f_{\text {ei } \alpha} \overline{l_{i L}} e_{\alpha R} \phi+h_{\alpha \beta} \overline{\left(\nu_{\alpha R}\right)^{c}} e_{\beta R} \zeta
$$

The extra singlets $Y_{a}$ and $Z$ get very large masses from the vev of the scalar $\chi$ and they donot couple with the light neutrinos. Since they are decoupled from the low energy sector, we shall not discuss them from now on. Although we have to introduce these singlet fields for purpose of anomaly cancellation, if one can implement this mechanism in a larger theory like grand unified theory or string theory, one may not require to introduce these fields. The scalar $\zeta$ give radiative mass splitting to neutrinos of different generations, which in turn gives neutrino flavour mixing.

The first term in this equation gives small Dirac mass to the neutrinos

$$
M_{\nu}=f_{\nu i \alpha}<\eta>
$$

We now assume that the Dirac mass matrix is diagonal and degenerate and to explain the hot component of the dark matter the diagonal elements are given by $f_{\nu i \alpha} \delta_{i \alpha}=m$. The neutrino Dirac mass matrix then becomes

$$
M_{\nu}=\left(\begin{array}{ccc}
m & 0 & 0 \\
0 & m & 0 \\
0 & 0 & m
\end{array}\right) .
$$

We further assume that in this basis the Yukawa interactions of the field $\zeta$ is also diagonal, but the charge lepton mass matrix is not diagonal. In general, it may be possible to diagonalise the charge lepton mass matrix and 
the neutrino mass matrix simultaneously, since the neutrino mass matrix is degenerate. However, the coupling of the charged scalar $\zeta$ will give radiative correction, which will break the mass degeneracy and will not allow to make the charged current interaction diagonal. As a result all the observed mixing may come from the charge lepton mass matrix and we can only determine them from experiments. The mixing angle will depend on the amount of radiative mass splitting.

In general, the charged lepton mass matrix can be diagonalised by a biunitary transformation

$$
V_{i k}^{\dagger} M_{e i \alpha} U_{\alpha \beta}=M_{e k \beta}^{\operatorname{diag}} \delta_{k \beta}
$$

Then the matrix $V_{i k}$, which diagonalises the matrix $M_{e i \alpha} M_{e j \alpha}^{\dagger}$, will enter in the charged current interactions. In the basis $\left[e_{i L}, e_{\alpha R}\right]$, in which the charged lepton mass matrix is diagonal, the charge current interaction of the neutrinos and the charged leptons will be given by,

$$
\mathcal{L}_{c c}=\overline{\nu_{i L}} \gamma_{\mu} V_{k i}^{\dagger} e_{k L} W^{\mu}
$$

The charged lepton mixing matrix $V_{i j}$ will introduce neutrino flavour mixing after the radiative mass splitting.

We now define the basis for the neutrinos $\left[\nu_{i L}^{e}, \nu_{\alpha R}^{e}\right]$, which has diagonal charged current interaction and are given by,

$$
\nu_{i L}^{e}=V_{i k} \nu_{k L} \quad \text { and } \quad \nu_{\alpha R}^{e}=U_{\alpha \beta}^{\nu} \nu_{\beta R}
$$

In this basis, the mass matrix is not diagonal. But when the mass matrix is diagonal and degenerate, we can always make transformations to the right handed fields and make them diagonal. In the basis $\left[\nu_{i L}^{e}, \nu_{\alpha R}^{e}\right]$, the neutrino mass matrix is given by,

$$
M_{\nu i j}^{e}=V_{i j} M_{\nu}
$$


But $V_{i j}$ commutes with $M_{\nu}$ and hence we can make a transformation $\nu_{\alpha R}^{e}=$ $V_{\beta \alpha}^{\dagger} \nu_{\beta R} ; \quad V_{\beta \alpha}=V_{i j}$, and diagonalise the mass matrix. However, in the presence of the radiative corrections due to $\zeta$, this is not possible.

We assume that the couplings of $\zeta$ to the right handed leptons, $h_{\alpha \beta}$, to be diagonal. Although the phenomenology of such dilepton have not been studied, one can extend the analysis of ref. [11] to constrain the parameters. If, in addition, we assume that $h_{11}<10^{-5}$, then there is only one constraint from the $(g-2)_{\mu}$, which is $h_{22}>0.3$ for $m_{\zeta} \sim 100 \mathrm{GeV}$. For $h_{33}$ there is no bound in this scenario and we can consider this to be of the order of 1 . With this choice we get a self energy radiative correction (with the internal loop containing charged leptons and $\zeta$ ) to the neutrino mass matrix in the basis $\left[\nu_{i L}, \nu_{\alpha R}\right]$, in which the charged current interaction is not diagonal but the $\zeta$ couplings are diagonal, given by

$$
M_{\nu i \alpha}=\left(\begin{array}{ccc}
m+m_{1}^{\zeta} & 0 & 0 \\
0 & m+m_{2}^{\zeta} & 0 \\
0 & 0 & m+m_{3}^{\zeta}
\end{array}\right)
$$

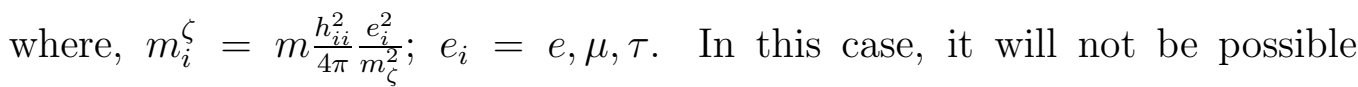
to diagonalise the neutrino mass matrix in the basis in which the charged current interaction is diagonal. Including the standard model self energy radiative corrections [12], we can now write down the neutrino mass matrix in the basis $\left[\nu_{i L}^{e}, \nu_{\alpha R}^{e}\right]$ as follows,

$$
M_{\nu i \alpha}^{e}=V_{i j} M_{\nu i \alpha}+\operatorname{diag}\left[\begin{array}{lll}
m_{1}^{e w} & m_{2}^{e w} & m_{3}^{e w}
\end{array}\right] V_{i j} M_{\nu j \alpha}
$$

where, $m_{i}^{e w}=\alpha_{w}\left(m_{e_{i}}^{2} / m_{w}^{2}\right), \quad e_{i} \equiv e, \mu, \tau$. This mass matrix can now be diagonalised to get the flavour mixing matrix and the mass ssquared difference between the different flavours of neutrinos. For any arbitrary choice of the mixing matrix it is difficult to solve this analytically. So, for purpose of illustration we demonstrate with a two generation example, and then present some realistic numbers for a three generation scenario which we check numerically. 
We consider the $\mu$ and $\tau$ family in the two generation example. For $V_{i j}=\left(\begin{array}{cc}\cos \theta & -\sin \theta \\ \sin \theta & \cos \theta\end{array}\right)$, the mass matrix is given by,

$$
M_{\nu i \alpha}^{e}=\left(\begin{array}{cc}
\left(m+m_{2}^{\zeta}\right)\left(1+m_{2}^{e w}\right) \cos \theta & -\left(m+m_{3}^{\zeta}\right)\left(1+m_{2}^{e w}\right) \sin \theta \\
\left(m+m_{2}^{\zeta}\right)\left(1+m_{3}^{e w}\right) \sin \theta & \left(m+m_{3}^{\zeta}\right)\left(1+m_{3}^{e w}\right) \cos \theta
\end{array}\right)
$$

which can be diagonalised with a bi-unitary transformation. The unitary matrix, which diagonalises $M_{\nu i \alpha}^{e}\left(M_{\nu j \alpha}^{e}\right)^{\dagger}$, gives the neutrino flavour mixing and is given by,

$$
V_{i j}^{e}=\left(\begin{array}{ccc}
\cos \phi & \sin \phi \\
\sin \phi & \cos \phi
\end{array}\right)
$$

where,

$$
\phi \sim \frac{1}{2} \tan ^{-1}\left[2 \cos \theta \sin \theta \frac{\left(m_{3}^{\zeta}-m_{2}^{\zeta}\right)}{\left(m_{3}^{\zeta}-m_{2}^{\zeta}\right)+\left(m_{3}^{e w}-m_{2}^{e w}\right)}\right]
$$

and the mass squared difference is given by,

$\left(m_{3}^{2}-m_{2}^{2}\right)^{2}=\left[2 m \cos \theta \sin \theta\left(m_{3}^{\zeta}-m_{2}^{\zeta}\right)\right]^{2}+\left[m\left(m_{3}^{\zeta}-m_{2}^{\zeta}\right)+m\left(m_{3}^{e w}-m_{2}^{e w}\right)\right]^{2}$.

It is clear from the expressions that both the electroweak radiative correction as well as the radiative corrections due to $\zeta$ will contribute to the mass squared difference. If $\zeta$ does not contribute to the mass difference, the mixing angle vanishes as pointed out earlier. For the mixing angle to be maximal we require the contribution of $\zeta$ to be of the same order or more than the contribution from the electroweak radiative corrections.

The mass difference generated by the standard model self energy radiative corrections (considering the Dirac mass of the neutrinos to be around $7 \mathrm{eV}$ ) are given by,

$$
\begin{aligned}
\Delta m_{\text {sol }} & =\left(m_{2}^{e w}\right)^{2}-\left(m_{1}^{e w}\right)^{2}=\alpha_{w} m^{2} \frac{m_{\mu}^{2}-m_{e}^{2}}{m_{w}^{2}}=0.6 \times 10^{-5} \\
\Delta m_{a t m} & =\left(m_{3}^{e w}\right)^{2}-\left(m_{2}^{e w}\right)^{2}=\alpha_{w} m^{2} \frac{m_{\tau}^{2}-m_{\mu}^{2}}{m_{w}^{2}}=1.4 \times 10^{-3}
\end{aligned}
$$


The $\nu_{e}-\nu_{\mu}$ mass difference $\Delta m_{\text {sol }}^{e w}$ is just enough to solve the solar neutrino problem, while the $\nu_{\mu}-\nu_{\tau}$ mass difference $\Delta m_{\text {atm }}^{e w}$ falls within the solution suggested by the recent super kamiokande result. If the contribution due to $\zeta$ are of the same order of magnitude (which is the case for $m_{\zeta} \sim 100$ $\mathrm{GeV}$ and $h_{11}=0, h_{22} \sim 0.1$ and $\left.h_{33} \sim 1\right)$, then we get the maximal mixing angle for the $\nu_{\mu}$ and $\nu_{\tau}$ oscillations and the relevant mass squared difference as required by the super kamiokande experiment. For the solar neutrino problem, the mass squared difference between $\nu_{e}$ and $\nu_{\mu}$ is just right for the MSW solution [9], but we only get the small mixing solution. In this scenario it will be difficult to explain the vacuum oscillation solution of the solar neutrino problem, since although one can adjust the parameters of the couplings of $\zeta$ to get a small radiative corrections, one cannot change the electroweak radiative correction. As a result, the mass squared difference between $\nu_{e}$ and any other neutrinos will be larger than required, unless there are new sterile neutrinos, to which the $\nu_{e}$ oscillates. Since the neutrino masses arise from a different higgs doublet, the neutrino mass mixing is not related to the CKM quark mixing matrix.

In summary, we pointed out that if neutrinos are Dirac particles, then we may start with a degenerate diagonal mass matrix with the diagonal elements to be around a few eV, so that neutrinos could be the hot dark matter of the universe. We also presented a model in which neutrinos could be light Dirac particles naturally. The mass degeneracy is broken by self energy radiative corrections, which can then allow enough mixing of these neutrinos to solve the atmospheric neutrino and solar neutrino problems. The standard model radiative corrections give a lower bound on the mass squared difference which is just enough to solve both the atmospheric neutrino and solar neutrino problems. 


\section{ACKNOWLEDGEMENT}

I would like to thank Profs. David Cline, Prof. D.P. Roy and M. Raidal for discussions. I would also like to thank Prof W. Buchmuller and the Theory Division, DESY, Hamburg for hospitality. Financial support from the Alexander von Humboldt Foundation is acknowledged. 


\section{References}

[1] H.V. Klapdor-Kleingrothaus, in Proc Lepton and Baryon number violatoin, Trento, April 1998; M. Günther et al, Phys. Rev. D 55 (1997) 54; L. Baudis et al, Phys. Lett. B 407 (1997) 219.

[2] J. Engel, S. Pittel and P. Vogel, Int. J. Mod. Phys. E 1 (1992) 1; A.N. Taylor, M. Rowan-Robinson, Nature 359 (1992) 396; M. Davis, F.J. Summers and D. Schlegel, Nature 359 (1992) 393.

[3] R. Davis, Prog. Part. Nucl. Phys. 32 (1994) 13; Y. Fukuda et al, Phys. Rev. Lett. 77 (1996) 1683; P. Anselmann et al, Phys. Lett. B 357 (1995) 237; B 361 (1996) 235.

[4] T. Kajita, Talk presented at Neutrino 1998 Conference; Y. Fukuda et al, hep-ex/9805006, hep-ex/9805021 and hep-ph/9807003.

[5] Particle Data Group, Eur. Phys. J. C 3 (1998) 1.

[6] R. Armbruster et al, Phys. Rev. C 57 (1998) 3414.

[7] A. Athanassopoulos et al (LSND Collaboration), Phys. Rev. Lett. 77 (1996) 3082, nucl-ex/9706006 and nucl-ex/9709006.

[8] S.T. Petcov and A. Yu. Smirnov, Phys. Lett. B322 (1994) 109; A. Joshipura, Z. Phys. C 64 (1994) 31; D.O. Caldwell and R.N. Mohapatra, Phys. Rev. D 50 (1994) 3477; D.G. Lee and R.N. Mohapatra, Phys. Lett. B 229 (1994) 463; A. Joshipura, Phys. Rev. D 51 (1995) 1321.

[9] L. Wolfenstein, Phys. Rev. D 17 (1978) 2369; S.P. Mikheyev and A. Yu. Smirnov, Yad. Fiz. 42 (1995) 1441 [Sov. J. Nucl. Phys. 42 (1985) 913].

[10] E. Ma and U. Sarkar, Phys. Rev. Lett. 80 (1998) 5716.

[11] F. Cuypers and S. Davidson, Eur. Phys. J. C 2 (1998) 503.

[12] L. Wolfenstein, Nucl.Phys. B 186 (1981) 147; U. Sarkar, Phys. Rev. D 35 (1987) 1528. 